\title{
Anomalous Spectral Types and Intrinsic Colors of Young Stars
}

\author{
Mark J. Pecaut \\ Rockhurst University, 1100 Rockhurst Rd., Kansas City, MO 64110 \\ email: mark. pecaut@rockhurst.edu
}

\begin{abstract}
We highlight differences in spectral types and intrinsic colors observed in pre-main sequence (pre-MS) stars. Spectral types of pre-MS stars are wavelength-dependent, with nearinfrared spectra being 3-5 spectral sub-classes later than the spectral types determined from optical spectra. In addition, the intrinsic colors of young stars differ from that of main-sequence stars at a given spectral type. We caution observers to adopt optical spectral types over nearinfrared types, since Hertzsprung-Russell (H-R) diagram positions derived from optical spectral types provide consistency between dynamical masses and theoretical evolutionary tracks. We also urge observers to deredden pre-MS stars with tabulations of intrinsic colors specifically constructed for young stars, since their unreddened colors differ from that of main sequence dwarfs. Otherwise, $V$-band extinctions as much as $\sim 0.6$ mag erroneously higher than the true extinction may result, which would introduce systematic errors in the H-R diagram positions and thus bias the inferred ages.
\end{abstract}

Keywords. stars: pre-main sequence, stars: fundamental parameters, starspots, open clusters and associations: individual ( $\eta$ Cha Cluster, TW Hydra Association, $\beta$ Pictoris moving group, Tucana-Horologium moving group)

\section{Introduction}

Two of the most fundamental parameters of a star - the effective temperature $\left(\mathrm{T}_{\text {eff }}\right)$ and luminosity - are based on simple, easy-to understand data such as the spectral type, extinction, and bolometric corrections. Determining these should be relatively error-free, right? Experience has taught observers that special care must be taken when characterizing pre-main sequence (pre-MS) stars, as these young stars require more attention than that of main-sequence dwarfs.

When observers desire to characterize a star's properties, they normally start with the spectral type. The spectral type of the star is determined by comparing characteristics of the spectrum with spectral standard stars. In doing this we can obtain an estimate of the temperature and a gross estimate of the surface gravity of the star. To quantify the extinction and reddening, the observer will compare the target star's observed colors to tabulated intrinsic colors of stars of the same spectral type to determine a color excess and use a total-to-selective extinction ratio value to estimate the extinction. Once the extinction has been quantified, a distance can be estimated (if not known), by assuming an age and consulting a theoretical isochrone, or assuming the star is on the mainsequence and calculating a main-sequence distance. Alternatively, if a trigonometric or kinematic parallax is known, we may compute the luminosity of the star. Normally, the point of these calculations is to place the star on the Hertzsprung-Russell (H-R) diagram and compare it to theoretical evolutionary models to estimate an age and mass.

Though this process seems fairly straightforward, there are many assumptions and systematic effects which can creep in and result in systematic errors in the fundamental parameters, such as temperature and luminosity (which are relatively model-free), and 
therefore the parameters derived from the evolutionary models. Assuming we are able to determine the spectral type with perfect fidelity, there are uncertainties in the tables which relate the spectral type, $\mathrm{T}_{\text {eff }}$, intrinsic color and bolometric correction. There are also many different varieties of such tables, with slight variations in their intrinsic colors, underlying temperature scale, and bolometric corrections. Some tables even contain selfinconsistent values for the bolometric corrections and the bolometric luminosity of the Sun (Torres 2010) †!

For populations of young stars this presents many problems because systematic errors in the fundamental properties of young, pre-MS stars will propagate into systematic errors in masses and ages (see Soderblom et al. 2014 for a full discussion of ages of young stars). This can skew the inferred evolutionary lifetime of gas-rich disks. Since gas giant planets can only form when gas is present in the circumstellar disk, these ages are also used to constrain giant planet formation timescales. Problems are also present when individual stars are mischaracterized. If a young star hosts a directly-imaged substellar object, the mass of the substellar object is estimated by comparing the luminosity and assumed age of the object with evolutionary models. Systematic effects in assumed ages can then propagate to wrong assumptions about the model-derived masses (e.g., $\kappa$ And b; Hinkley et al. 2013; Bonnefoy et al. 2014), which may misdirect planet formation theories. Thus, systematic errors in individual parameters, such as spectral types and intrinsic colors, can propagate down and fundamentally limit our ability to test star and planet formation theories.

\section{Spectral Types}

The spectral type of a target is one of the most useful measurements, since many other stellar properties are usually derived with some dependence on the spectral type. Young stars, like most stars, are typed by comparing their spectra with that of spectral standards, and this has historically been performed using optical spectra. However, many low-mass stars are brighter in the near-infrared (NIR) and so it seems completely reasonable to perform this same measurement with NIR spectra as well. Two interesting cases are that of TW Hya and V4046 Sgr.

TW Hya, one of the most well-studied classical T-Tauri stars and a member of the youngest nearby moving group that bears its name (the TW Hydra Association), has typically been assigned a temperature type of K7 using optical spectra (K8IVe, Pecaut \& Mamajek 2013; K6Ve, Torres et al. 2006; K6e, Hoff et al. 1998; K7e, de la Reza et al. 1989; K7 Ve, Herbig 1978). However, Vacca \& Sandell (2011) assigned a type of M2.5V using NIR spectra from SpeX, which implied a very young age of $\sim 3$ Myr instead of the much older, more often quoted age of $\sim 10$ Myr (Barrado Y Navascués 2006). This discrepancy is a source of great confusion - which spectral type should one adopt when characterizing young stars?

V4046 Sgr, a young binary member of the $\beta$ Pictoris moving group harboring a gasrich disk of its own, has also been typed in both the optical and NIR and the same effect is observed - the NIR spectral type is about 3-5 subtypes later than the optical spectral type (Kastner et al. 2015). However, unlike TW Hya, V4046 Sgr has dynamical mass constraints from radial velocities and gas dynamics (Rosenfeld et al. 2012). Kastner et al. (2015) have placed these two young stars on the H-R diagram assuming the optical spectral types in one case and the NIR spectral types in another case. Comparing these

$\dagger$ There are even a variety of values used for the bolometric magnitude of the Sun. Here we adopt $M_{b o l, \odot}=4.7554 \pm 0.0004$, as advocated in Mamajek (2012). 
two sets of H-R diagram positions with theoretical evolutionary models, the NIR spectral types are inconsistent with the dynamical mass constraints and Kastner et al. (2015) thus urged caution against using the NIR spectral types on young stars.

Stauffer et al. (2003) have studied this wavelength-dependent spectral type effect among the zero-age main sequence $\mathrm{K}$ dwarfs in the Pleiades ( 135 Myr; Bell et al. 2014). The Stauffer et al. (2003) study found that the spectral type of Pleiades K-type stars were systematically $\sim 1$ subtype later in the red optical spectra than the blue optical spectra. Furthermore, they did not observe this spectral type anomaly in members of the older Praesepe cluster ( 650-800 Myr; Gáspár et al. 2009; Bell et al. 2014; Brandt \& Huang 2015). Stauffer et al. (2003) argued that spots were a major factor in this effect, and concluded that there must be more than one photospheric temperature present in the Pleiades K-dwarfs. Pre-main sequence stars are magnetically very active as well, and observations indicate large filling factors on their surfaces (Berdyugina 2005), consistent with this effect.

\section{Intrinsic Colors}

Many studies in the past two decades have pointed out that stellar intrinsic colors of young stars are different than that of typical main sequence dwarfs. Gullbring et al. (1998) had noted this for both classical and weak-lined T-Tauri stars in Taurus. Da Rio et al. (2010) have noted this in the Orion Nebula Cluster (ONC) and dereddened the very young ONC cluster members using a specially constructed spectral-type color sequence for young stars. However, their spectral type-color sequence was not published as part of their study. Luhman (1999) and Luhman et al. (2010b,a) went much further and constructed a color-spectral type sequence for pre-MS late K- and M-type stars and brown dwarfs. Most recently, the Pecaut \& Mamajek (2013) study released a comprehensive tabulation of the intrinsic colors for pre-MS stars from F-type to late M-type with the most popular photometric bands - Johnson-Cousins $B V I_{C}, 2 \mathrm{MASS} J H K_{S}$ and the recently available WISE $W 1, W 2, W 3$ and $W 4$ bands. In addition, we fit the observed spectral energy distributions to Phoenix-NextGen synthetic spectra (Allard et al. 2012) to infer an effective temperature $\left(\mathrm{T}_{\text {eff }}\right)$ and bolometric correction $(\mathrm{BC})$ scale for pre-MS stars. This young spectral type- color- $\mathrm{T}_{\text {eff }}-\mathrm{BC}$ scale was constructed using all the known (as of July 2013) members of the nearest moving groups - the $\eta$ Cha Cluster, the TW Hydra Association (TWA), the $\beta$ Pictoris moving group and the Tucana-Horologium (Tuc-Hor) moving group.

Two color-color plots for young stars are shown in Figure 1 . We plot $V-K_{S}$ on the horizontal axis as a proxy for $\mathrm{T}_{\text {eff }}$ against $J-H$ (left) and $K_{S}-W 1$ (right). The stars are predominantly clustered around the dwarf and giant sequence until the two diverge around $V-K_{S}$ of $\sim 4$, where they lie between the dwarf and giant locus. This strongly suggests surface gravity is a major factor in the deviation of intrinsic colors from that of dwarfs, since we expect that pre-MS stars will have surface gravities somewhere between that of dwarfs and giants.

An indication of the importance of accounting for the difference between pre-MS colors and dwarf colors is shown in Figure 2a. The $J-H$ colors of young stars are significantly redder than the dwarf colors at a given spectral type. If $J-H$ dwarf colors were used to estimate extinction for an unreddened M0 star, it would erroneously appear to have an $A_{V} \simeq 0.6 \mathrm{mag}$ of extinction! If dwarf colors are used to deredden a population of young stars, a systematic bias may be introduced, depending on the particular color used, which may cause their luminosities to be systematically overestimated and thus their ages would be systematically underestimated. 

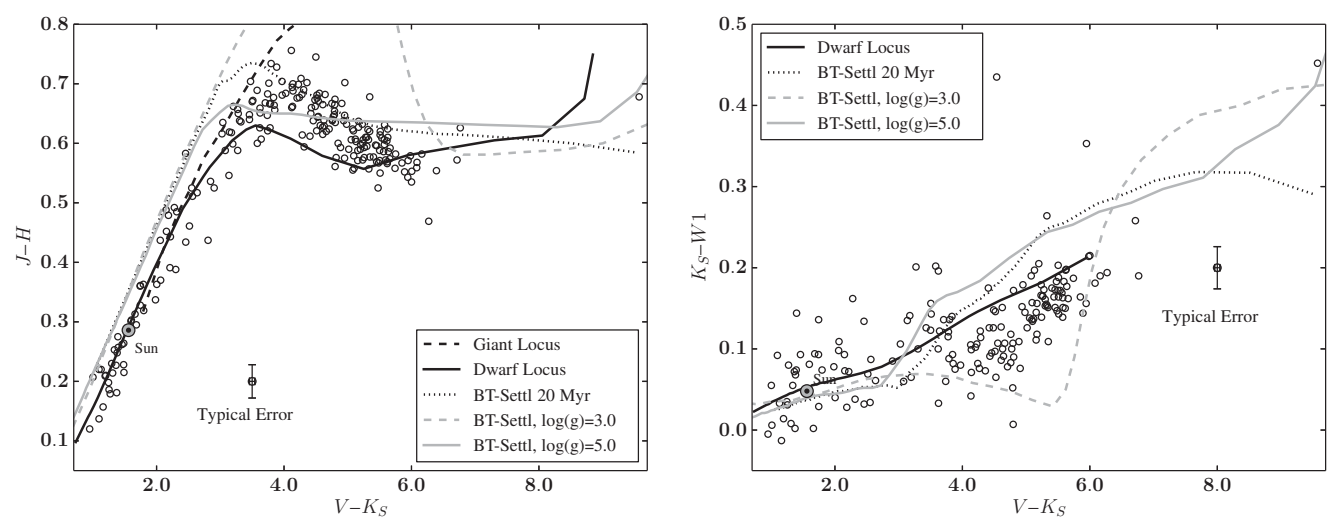

Figure 1. $V-K_{S}$ versus $J-H$ (left) and $V-K_{S}$ versus $K_{S}-W 1$ (right) for young stars in the $\eta$ Cha cluster, the TW Hydra Association, the $\beta$ Pictoris moving group and the Tucana-Horologium moving group from the sample in Pecaut \& Mamajek (2013), with the addition of 129 new Tuc-Hor members from Kraus et al. (2014). The dwarf locus is adopted from Pecaut \& Mamajek (2013) and the giant locus is adopted from Bessell et al. (1998). 20 Myr isochronal colors are constructed by adopting surface gravities from a Baraffe et al. (1998) 20 Myr isochrone with synthetic colors from the BT-Settl models of Allard et al. (2012).
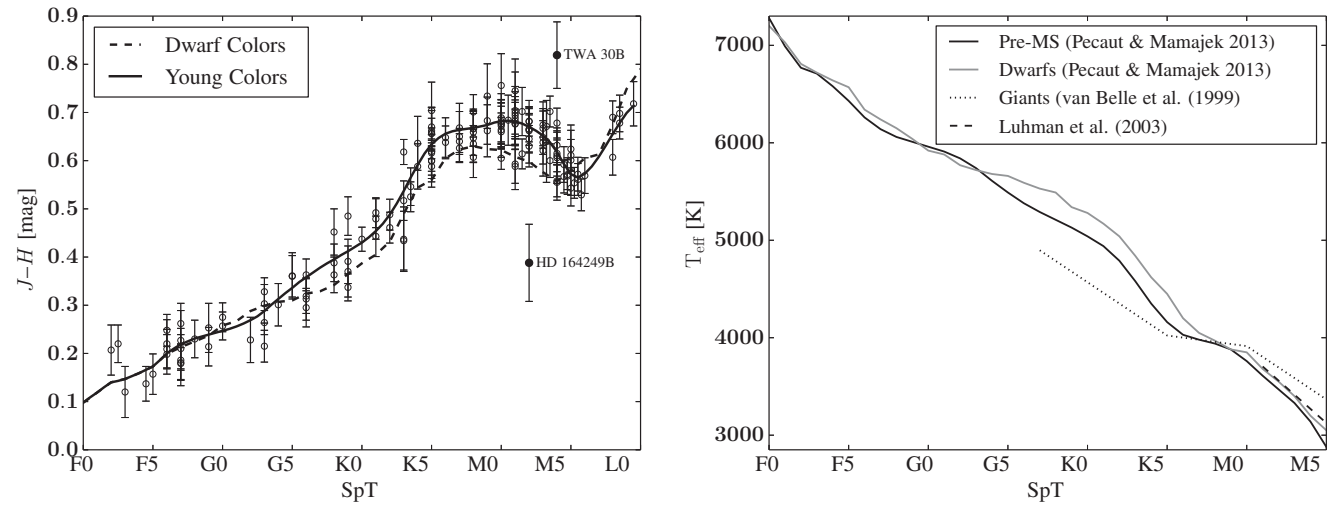

Figure 2. Left: Spectral type versus $J-H$ for young stars in the $\eta$ Cha cluster, the TW Hydra Association, the $\beta$ Pictoris moving group and the Tucana-Horologium moving group from the sample in Pecaut \& Mamajek (2013), with the addition of 129 new Tuc-Hor members from Kraus et al. (2014). Right: Spectral type versus effective temperature for pre-MS stars (Pecaut \& Mamajek 2013), dwarf stars (Pecaut \& Mamajek 2013), giants (van Belle et al. 1999), and the M-type $T_{\text {eff }}$ scale from Luhman et al. (2003). The pre-MS temperature scale shown is $\sim 200 \mathrm{~K}$ cooler than the dwarf scale for types $\sim \mathrm{G} 5-\mathrm{K} 5$.

A careful look at the temperature scale of pre-MS stars is warranted, since a star's adopted spectral type is used to infer the $\mathrm{T}_{\text {eff }}$. A frequently used temperature scale for M-type stars is the scale of Luhman et al. (2003), which is intermediate between dwarfs and giants. However, in the past few years two important developments have been made available: (1) with the release of the 2MASS and WISE catalogs (Skrutskie et al. 2006; Cutri et al. 2012), photometry is available which covers large sections of the star's spectral energy distributions (SED) and (2) high-quality synthetic spectra are available from the Phoenix-NextGen group (Allard et al. 2012) which include updated molecular opacities and model low-temperature stars and brown dwarfs more successfully than ever possible previously. Thus it is possible to obtain tight constraints on the $\mathrm{T}_{\text {eff }}$ of an individual unreddened pre-MS member of one of the nearby, young moving groups through fitting 
the observed SED with synthetic models. Pecaut \& Mamajek (2013) fit Phoenix-NextGen BT-Settl models of Allard et al. (2012) to the observed SEDs of the members of $\eta$ Cha, TWA, $\beta$ Pic and Tuc-Hor to tie their updated spectral type intrinsic color tabulation to a $T_{\text {eff }}-\mathrm{BC}$ system. The results of this $\mathrm{T}_{\text {eff }}$ scale are shown in Figure $2 \mathrm{~b}$. Pre-MS stars are $\sim 200 \mathrm{~K}$ systematically cooler than their main sequence counterparts at a given spectral type, for spectral types $\sim \mathrm{G} 5-\mathrm{K} 5$.

\section{Conclusions}

When characterizing the basic observations and properties of young stars, such as spectral type, $\mathrm{T}_{\text {eff }}$, reddening and extinction, care must be exercised to avoid systematic errors and biases. It is important that optical spectra be used to constrain the spectral type of the star, since these seem to best represent the effective temperature of the stars. Some evidence points to spots as a factor in wavelength-dependent spectral types, discussed in detail by Gullbring et al. (1998) and Stauffer et al. (2003).

When considering or using tabulations of intrinsic colors, it is important to adopt intrinsic colors for pre-MS stars when the stars are still contracting to the main sequence. Comparisons with dwarf and giant colors as well as theoretical synthetic spectra of different surface gravity points to surface gravity as an important effect altering the intrinsic colors of pre-MS stars. If individual extinctions are mis-estimated using dwarf colors, stars placed on the H-R diagram may have their luminosities systematically overestimated and the population may appear younger simply because observers are unable to account for reddening and extinction properly. This clearly inhibits our ability to accurately test theoretical evolutionary models and may systematically bias ages, which has consequences reaching down to mis-estimating age spreads in star-forming regions, underestimating the evolutionary timescales of disks and planet formation timescales, and systematic errors when inferring the initial mass function (IMF) in pre-MS stellar populations.

\section{References}

Allard, F., Homeier, D., \& Freytag, B. 2012, Royal Society of London Philosophical Transactions Series A, 370, 2765

Baraffe, I., Chabrier, G., Allard, F., \& Hauschildt, P. H. 1998, A\&SA, 337, 403

Barrado Y Navascués, D. 2006, A\&A, 459, 511

Bell, C. P. M., Rees, J. M., Naylor, T., et al. 2014, MNRAS, 445, 3496

Berdyugina, S. V. 2005, Living Reviews in Solar Physics, 2, 8

Bessell, M. S., Castelli, F., \& Plez, B. 1998, A\&A, 333, 231

Bonnefoy, M., Currie, T., Marleau, G.-D., et al. 2014, A\&A, 562, A111

Brandt, T. D., \& Huang, C. X. 2015, ArXiv e-prints, arXiv:1504.00004

Cutri, R. M., Skrutskie, M. F., van Dyk, S., et al. 2012, VizieR Online Data Catalog, 2281, 0

Da Rio, N., Robberto, M., Soderblom, D. R., et al. 2010, ApJ, 722, 1092

de la Reza, R., Torres, C. A. O., Quast, G., Castilho, B. V., \& Vieira, G. L. 1989, ApJ, 343, L61

Gáspár, A., Rieke, G. H., Su, K. Y. L., et al. 2009, ApJ, 697, 1578

Gullbring, E., Hartmann, L., Briceno, C., \& Calvet, N. 1998, ApJ, 492, 323

Herbig, G. H. 1978, Can Post-T Tauri Stars Be Found?, ed. L. V. Mirzoyan, 171

Hinkley, S., Pueyo, L., Faherty, J. K., et al. 2013, ApJ, 779, 153

Hoff, W., Henning, T., \& Pfau, W. 1998, A\&A, 336, 242

Kastner, J. H., Rapson, V., Sargent, B., Smith, C. T., \& Rayner, J. 2015, in Cambridge Workshop on Cool Stars, Stellar Systems, and the Sun, Vol. 18, Cambridge Workshop on Cool Stars, Stellar Systems, and the Sun, ed. G. T. van Belle \& H. C. Harris, 313-320

Kraus, A. L., Shkolnik, E. L., Allers, K. N., \& Liu, M. C. 2014, AJ, 147, 146 
Luhman, K. L. 1999, ApJ, 525, 466

Luhman, K. L., Allen, P. R., Espaillat, C., Hartmann, L., \& Calvet, N. 2010a, ApJS, 189, 353 —. 2010b, ApJS, 186, 111

Luhman, K. L., Stauffer, J. R., Muench, A. A., et al. 2003, ApJ, 593, 1093

Mamajek, E. E. 2012, ApJ, 754, L20

Pecaut, M. J., \& Mamajek, E. E. 2013, ApJS, 208, 9

Rosenfeld, K. A., Andrews, S. M., Wilner, D. J., \& Stempels, H. C. 2012, ApJ, 759, 119

Skrutskie, M. F., Cutri, R. M., Stiening, R., et al. 2006, AJ, 131, 1163

Soderblom, D. R., Hillenbrand, L. A., Jeffries, R. D., Mamajek, E. E.,\& Naylor, T. 2014, Protostars and Planets VI, 219

Stauffer, J. R., Jones, B. F., Backman, D., et al. 2003, AJ, 126, 833

Torres, C. A. O., Quast, G. R., da Silva, L., et al. 2006, A\&A, 460, 695

Torres, G. 2010, AJ, 140, 1158

Vacca, W. D. \& Sandell, G. 2011, ApJ, 732, 8

van Belle, G. T., Lane, B. F., Thompson, R. R., et al. 1999, AJ, 117, 521 